\title{
A Segmented Ring, Cylindrical Ion Trap Source for Time-of-Flight Mass Spectrometry
}

\author{
Qinchung Ji, Michael R. Davenport, and Christie G. Enke \\ Department of Chemistry, University of New Mexico, Albuquerque, New Mexico, USA \\ John F. Holland \\ Department of Biochemistry, Michigan State University, East Lansing, Michigan, USA
}

\begin{abstract}
An ion trap source has been designed for use with time-of-flight (TOF) mass analysis. Two thin diaphragms make up a segmented ring electrode; the end cap electrodes are planar wire mesh. The potential field produced by the rf voltage applied between the ring and end cap electrodes resembles that of the cylindrical ion trap. The trapped ion population for ions created by electron impact exhibits linear growth against a first-order loss that has a time constant of about $50 \mu \mathrm{s}$; no ion loss occurs when the electron beam is off. The observed value of $q_{z}$ at low-mass cutoff for rf ion storage is -0.84 . Pulsed extraction of all ions is accomplished by switching the trap electrodes from $\mathrm{rf}$ to voltages required to provide a linear dc extraction field. The TOF flight path includes a wide energy range reflectron. Better than unit mass resolution is achieved through $\mathrm{m} / z 500$ without collisional ion cooling. With an extraction rate of $1 \mathrm{kHz}$ and a recording rate of 4 spectra per second, a linear working curve is obtained between $36 \mathrm{pg}$ and $18 \mathrm{ng}$ of chlorobenzene delivered chromatographically. The system has demonstrated the potential to achieve a very high sample utilization efficiency at high spectral generation rates. (J Am Soc Mass Spectrom 1996, 7, 1009-1017)
\end{abstract}

$\mathrm{O}$ ne of the principal obstacles in the development of time-of-flight mass spectrometry (TOFMS) for detection and characterization of continuously introduced sample (as from a gas chromatograph) has been the low duty cycle of the traditional time-of-flight mass analyzer. Even with continuous ionization, unless some ion storage mechanism is employed, only the ions generated in the last few microseconds remain in the source at the time of extraction for TOF analysis. For a 200- $\mu$ s flight time and a corresponding $5-\mathrm{kHz}$ ion extraction frequency, this corresponds to a sample utilization efficiency of less than $1 \%$.

A solution to the duty-cycle problem of TOF-MS could have particular value in a variety of applications where the rapid spectral generation rate of TOF-MS is needed without compromising sensitivity. In particular, time-of-flight mass spectrometry with time-array detection (complete acquisition of every extraction spectrum) has been identified as having the potential to eliminate the tradeoffs among sensitivity, chromatographic resolution, and mass spectral information that confront all operators of chromatography-mass spectrometry instruments based on scanning mass analyzers [1]. Furthermore, TOF-MS is uniquely capable of providing the hundreds or even thousands of full mass

Address reprint requests to Christie G. Enke, Department of Chemistry, University of New Mexico, Albuquerque, NM 87131. spectra per second required to provide an adequate sampling rate for the high-speed chromatography now being developed. Finally, the high ion compaction of TOF-MS and the full ion utilization of time-array detection could provide a full sample utilization chromatographic detector of unprecedented sensitivity.

A logical choice for an ion storage device to improve the sample utilization efficiency for TOF-MS is the Paul quadrupole ion trap [2]. The principal problem in development of an ion trap to serve as a source for time-of-flight mass spectrometry resides in the difference between the shapes of the isopotential field lines required for optimum operation of the source during ion trapping and during ion extraction. The quadrupole ion trap was used as a source for time-offlight mass spectrometry as early as 1979 [3]. More recently, the application of time-of-flight analysis of trapped ions has reappeared in the form of an instrument for the analysis of ions generated externally by matrix-assisted laser desorption-ionization and by electrospray ionization [4]. In all of these works, the electrode geometry that creates the fields needed for ion trapping does not accommodate the unidirectional linear field essential for the time-of-flight ion optics to bring isomass ions to a good temporal focus at the detector. As the work in Lubman's laboratory has demonstrated, this problem can be greatly ameliorated by allowing the ions to be collisionally cooled to the center of the trap before extraction. From this position, 
the extraction field vectors for the ions are almost parallel and equal, and reasonable mass resolution can be obtained. However, this solution to the resolution problem is inconsistent with the goals of the present work because the required cooling step significantly reduces both the duty cycle for sample utilization and the maximum spectral generation rate.

Other workers in TOF-MS have employed a focused beam of ionizing electrons to form a shallow potential well for ion storage between the extractions $[5,6]$. In our experience, ion loss rate from such a well is affected adversely by the presence of carrier gas as encountered with chromatographic applications.

The source design we have chosen to implement most closely resembles a cylindrical ion trap. In the traditional cylindrical ion trap, the hyperbolic-shaped electrodes of the quadrupole ion trap are replaced by simpler shapes, namely planar end caps separated by a cylindrical ring electrode [7]. In our design [8], the cylindrical ring electrode is further approximated by two or more thin, circular diaphragms as shown in Figure 1. Two filaments that supply energetic electrons for electron impact ionization are located just outside the segmented ring electrode. The electrons are guided into the ion source through the slits in the electron entry electrodes located just above and below this segmented ring ion trap storage source (SRS). In the ionization and storage modes, the ring electrode elements both have the same rf potential and the end caps are grounded. This gives rise to a field that is approximately similar to the quadrupole ion trap and is effective for ion storage as shown in Figure 2a. During ion extraction, the rf is turned off and a unidirectional extraction field is achieved by application to

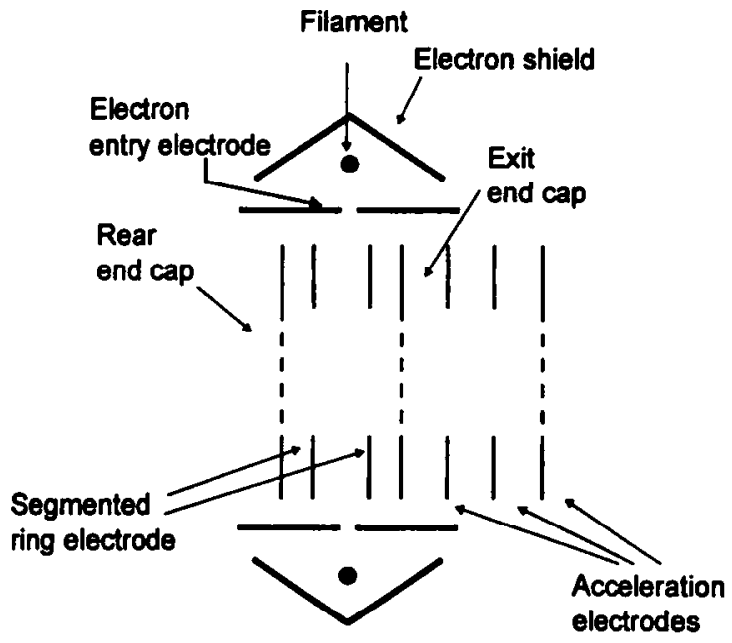

Figure 1. A cross section of the segmented ring ion trap source with electron impact ionization configuration. The end caps and ring electrodes are flat, annular rings made of solid metal. The open hole in the center of the end cap electrodes is covered with fine wire mesh. The two elements in the acceleration field region are open and mesh-covered rings, respectively. The electrons enter the ion source through the $1-\mathrm{mm} \times 1-\mathrm{cm}$ open slit of the electron entry electrodes on both sides.

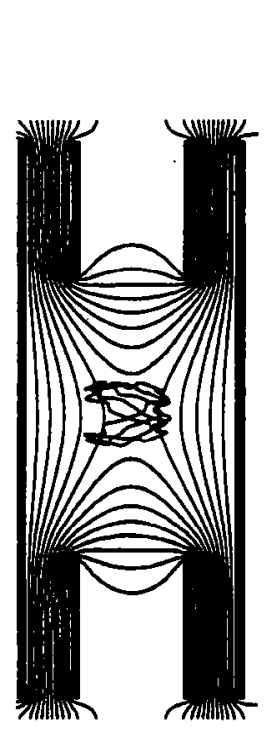

(a)

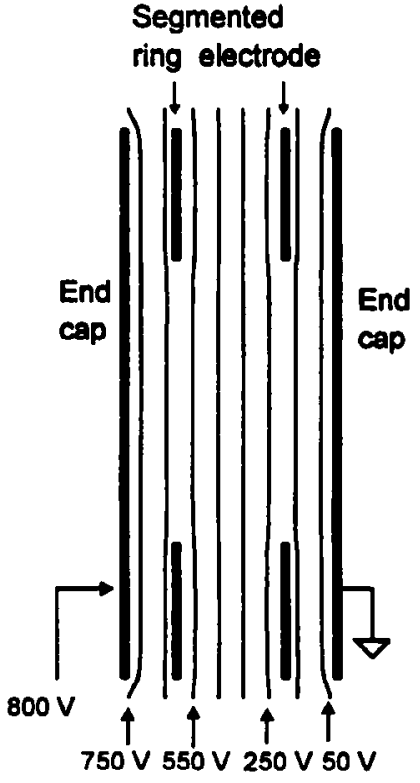

(b)
Figure 2. The equipotential contours for the segmented ring ion trap in the storage mode (a) and the extraction mode (b). A simulated ion trajectory $\left[\mathrm{m} / z 100 \mathrm{u}, V_{\mathrm{rf}}=600 \mathrm{~V}\right.$ (peak-to-peak), $\left.f_{\mathrm{rf}}=1.2 \mathrm{MHz}\right]$ is superimposed on the field lines in (a). In (b), The voltages applied to the segmented ring electrodes are proportional to their distance from the exit end cap. The nearly planar field lines are distorted only by the thickness of the ring electrodes.

the rear end cap and to each ring electrode element, a voltage proportional to its distance from the exit end cap electrode. The unidirectional extraction field is shown in Figure $2 b$.

Because of the considerable depth over which the ions will be located when the extraction field is applied, the extracted ions will have a wide distribution of kinetic energies along the flight path. Good temporal focusing of isomass ion packets at the detector is achieved through the use of an ion reflectron specially designed to cover an unusually wide range of ion energies [9]. The overall instrument design is shown in Figure 3. As demonstrated in the Results and Discus-

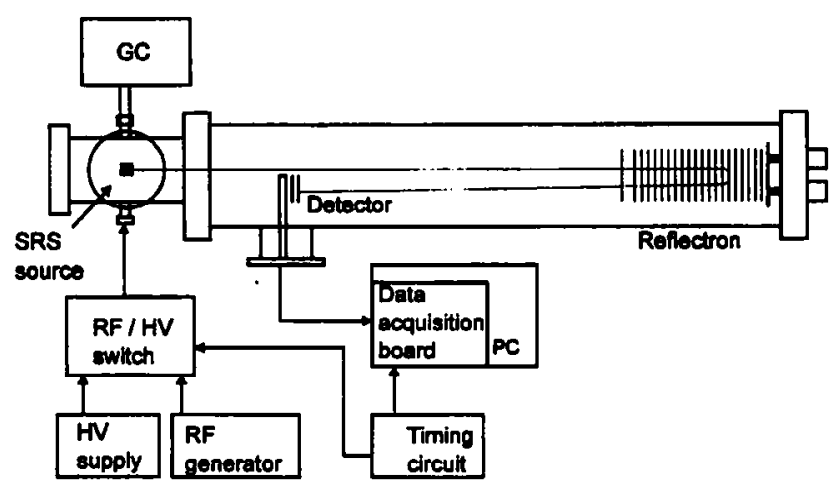

Figure 3. A block diagram of the time-of-flight instrument with segmented-ring ion trap source. For construction details, refer to the Experimental section. 
sion section, effective ion storage of continuously generated ions, mass resolution in excess of 1000 (full width at half maximum), and rapid spectral generation have been achieved. A critical part of this instrument is the high voltage switching circuitry that switches between the ionization-storage mode and the extraction function in only 45 ns.

The complete instrument described in this report can be viewed as either a trapping ion source for TOF-MS or as a TOF mass analyzer for an ion trap mass spectrometer. We refer to this instrument as a segmented ring source-time-of-flight (SRS-TOF) mass spectrometer.

\section{Experimental}

The time-of-flight mass spectrometer has a 10-in.-diameter, 58-in.-long flight chamber. The source chamber is a stainless steel four-way cross formed of 6-in.-i.d. tubes. The segmented ring ion trap storage source is mounted on a flange that attaches to one end of the four-way cross. As shown in Figure 1, the end caps and ring electrode are ring diaphragms with a 12.7$\mathrm{mm}$-i.d. hole at the center. The end caps are covered with stainless steel mesh ( $86 \%$ transmission); they are $9.4 \mathrm{~mm}$ apart. The center two rings are $2.5 \mathrm{~mm}$ apart. The source region is pumped by a Balzers (Hudson, $\mathrm{NH}$ ) TPH520 turbo pump and is separated from the remainder of the flight tube by a baffle through which a 1-in.-diameter and 8-in.-long tube provides an ion flight path while it limits the conductance between the source and flight chambers. A deflection plate is placed along the ion path. Ions are blocked from entering the flight chamber during the ionization period. This blockage reduces a large portion of the background ions that reach the detector. The flight chamber is pumped by another Balzers TPH520 turbo pump. The ion reflectron consists of 22 concentric rings with stainless steel screen ( $86 \%$ ion transmission efficiency) in the center of the first, second, and last reflectron rings. The ion reflectron is mounted on a flange bolted to the end of the flight tube. There are two mechanical feedthroughs for adjustment of the reflectron angle. The detector is mounted through a side flange located 52 in. from the reflectron end of the flight tube. The source and reflectron assemblies are made of components fabricated out of 304 stainless steel in machine shops at Michigan State University and the University of New Mexico. The flight tube and source housing are connected to earth ground. The flight path is maintained field-free at $1800 \mathrm{~V}$ by connection of the flight path voltage source to a steel mesh liner that is insulated from the flight tube by ceramic separators.

The ion detector is a chevron assembly of two 40-mm microchannel plates (Galileo Electro-Optics, Sturbridge, MA) operated with a voltage of -800 to $-980 \mathrm{~V}$ across each microchannel plate to achieve appropriate gain. The output signal is terminated by a $50-\Omega$ resistor at its connection to a Precision Instru- ments (Knoxville, TN) model 9825 transient recorder located in a Gateway 2000 (Sioux City, SD) P5-90 computer that provides data acquisition and data analysis functions.

Samples were introduced through a $0.1-\mathrm{mm}$-i.d., 10-m-long glass capillary column contained in a heated transfer line (Finuigan MAT, San Jose, CA) that emanates from a Hewlett-Packard (Avondale, PA) model 5890 chromatograph. Samples could be introduced either continuously from a helium-swept vial contained in the chromatographic oven or by injection through the chromatograph's injection port. In the latter case, a split injection system was used with a split ratio of 1:550. The chemicals used were perfluorotetrabutylamine (PFTBA; FC-43, Scientific Instrument Services, Ringoes, NJ), chlorobenzene (reagent grade, J. T. Baker Inc., Phillipsburg, NJ), and hexane [high performance liquid chromatography (HPLC) grade, EM Science, Gibbstown, NJ].

The static voltages for the reflectron, detector, and source are controlled manually. The electronic system that generates the $\mathrm{rf}$ voltage and the extraction voltage for the segmented ring source electrodes is shown in Figure 4. To obtain the highest resolution and intensity from the storage-extraction cycle it is important to find the best phase of rf (storage) cycle in which to initiate the extraction cycle and then maintain this ideal phase relationship for all experiments. This ideal timing for the SRS-TOF mass spectrometer begins with a common time base (Wavetek function generator, San Diego, CA, model 171) to drive the rf amplifier and the extraction timing. The TTL (transistor-transistor logic) signal from the function generator is routed through an adjustable delay to provide the optimum phase for all subsequent timing functions. This delayed clock is connected to a programmable modulus counter to generate an extraction clock signal with a frequency $1 / 128-1 / 16384$ that of the rf. The extraction clock signal triggers an ionization timer that enables electrons to enter the trap for an adjustable time from 6 to $600 \mu \mathrm{s}$. The end of the ionization time triggers the extraction delay timer with a range from 8 to $1000 \mu \mathrm{s}$. The end of this ion storage time triggers the ion extraction pulse generator. To preserve the desired rf phase-extraction timing relationship, the extraction trigger is synchronized with the delayed TTL clock signal by a HC74 D latch (National Semiconductor, Santa Clara, CA). The typical extraction trigger pulse width is $2 \mu \mathrm{s}$. The synchronized extraction trigger is buffered and routed to the data acquisition system, detector display, and high-speed, high-voltage switches attached to the disks of the segmented ring. Prior to the extraction trigger, a 1.2-MHz, rf signal adjustable from 200 to $900 \mathrm{~V}_{\mathrm{pp}}$ is applied to the segmented ring of the ion trap through a high-speed, high-voltage $\mathrm{N}$ channel MOSFET (metal-oxide-semiconductor fieldeffect transistor) assembly. At the command of the extraction trigger pulse, the rf side is turned off and the extraction voltage field-effect transistors (FETs) are 


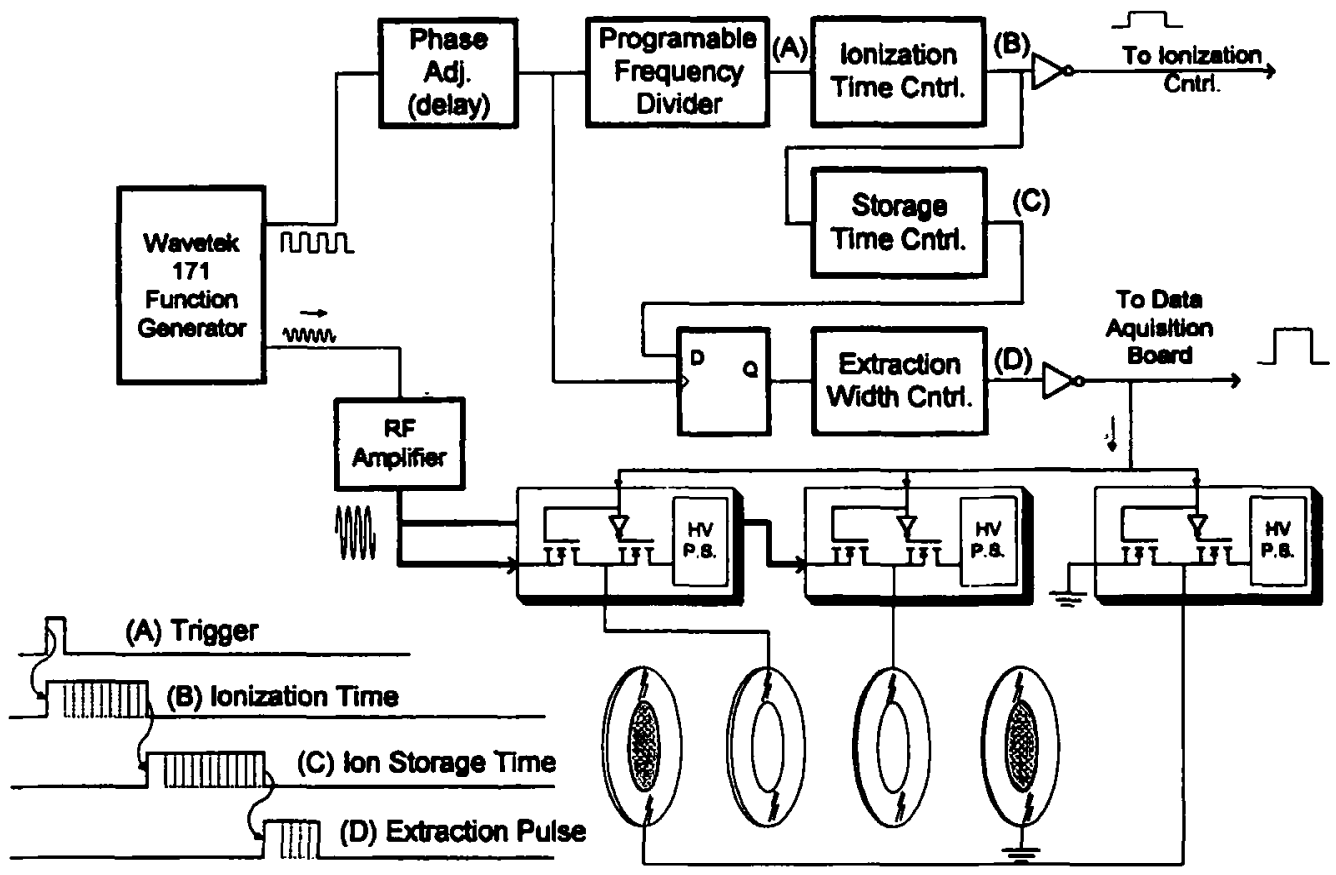

Figure 4. A block diagram of the switching and timing circuit that achieves a repetitive measurement cycle including ionization with storage (ionization time), storage without ionization (extraction delay time), and ion extraction.

turned on for the duration of the extraction pulse. At the falling edge of the extraction trigger, the extraction voltage MOSFET switches are turned off and the $\mathrm{rf}$ side is turned back on. All switch operation is breakbefore-make. Extraction voltages for the ring discs are supplied by individual 10-1200-V Spellman (Plainview, NY) multiphase power supplies. The rf is generated with a high-speed, high-power Apex (Tucson, AZ) PA09 op amp and the Hi-Q rf head from an early model Extrel (Pittsburgh, PA) quadrupole power supply.

\section{Results and Discussion}

In all the experiments described in this section, timeof-flight mass analysis was used to determine the mass-to-charge ratio values and abundances of the ions present in the trap at the time of extraction. The ionization conditions (filament offset relative to the end cap electrodes and filament current), the ionization time, the rf voltage, and the delay time between the cessation of ionization and ion extraction are under operator control. In the first part of this section, these variables are used to characterize the ion trap as an ion storage device; in the second part, the qualities of the time-of-flight mass analysis (resolution and sensitivity) are determined as a function of the source parameters.

\section{The Source as an Ion Trap}

The trapping characteristics of the segmented ring ion source were documented in a series of experiments designed to reveal the nature of ion accumulation during the ionization part of the cycle. A sample vial of chlorobenzene was placed in the gas chromatography oven with constant temperature $\left(25^{\circ} \mathrm{C}\right)$. Helium gas was introduced to this sample vial to carry the vapor of chlorobenzene into the ion source. This arrangement maintained a steady-state pressure of chlorobenzene in the source. The filament current was set to $6.5 \mathrm{~A}$. Ions were formed for various lengths of time after which the ions were immediately extracted and the abundances of the major ions $(\mathrm{m} / \mathrm{z} 112$ and 77) were determined. The results of this experiment are shown in Figure 5a. The ion abundances follow a consistent growth with increasing ionization time as discussed in subsequent text.

To separately determine the trapping efficiency and the nature of the ion loss, a series of experiments were performed in which an adjustable delay time was introduced between ion formation and ion extraction. In Figure 5b, the measured abundances of the chlorobenzene ions are shown as a function of the delay time after an ionization time of $142 \mu \mathrm{s}$. In this experiment, essentially no ion loss is observed. Repetition of this same experiment after an ionization time of $342 \mu$ s (Figure 5c) again shows no loss for periods up to $475 \mu \mathrm{s}$. From this experiment, it seems clear that the loss mechanism observed during ion formation is related to the ionization process, not to the ion storage mechanism.

The mass-to-charge ratio range over which ions will be trapped efficiently is dependent on the frequency and amplitude of the rf voltage as well as the dimensions of the ion trap [10]. The expected relationship is shown in eq 1, which is derived from the Matthieu 


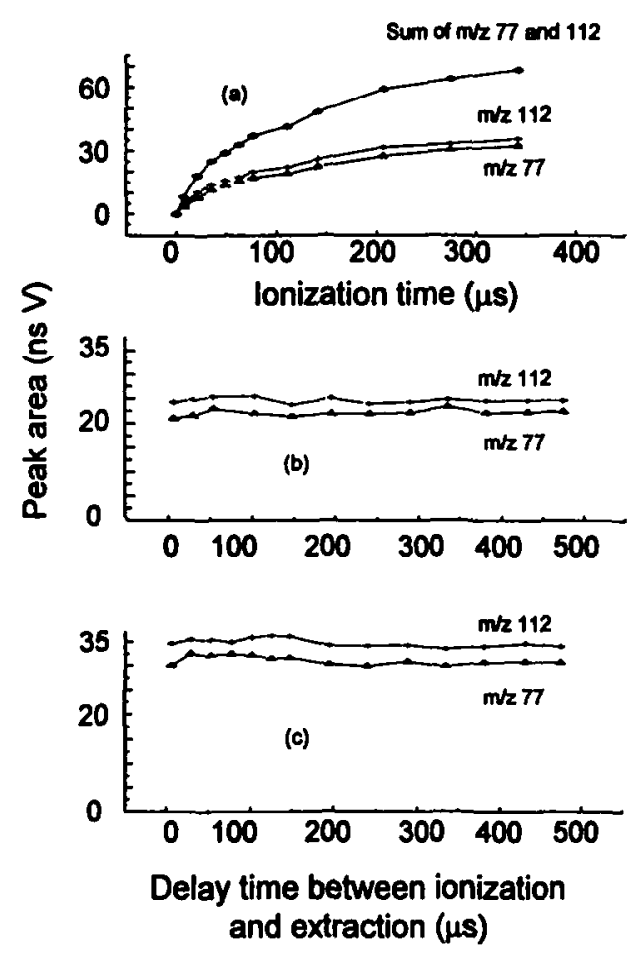

Figure 5. The abundance of the major ions of chlorobenzene as a function of (a) continuous ionization time and (b) extraction delays after an ionization time of $142 \mu \mathrm{s}$ and (c) $342 \mu \mathrm{s}$. The rf applied is $600 \mathrm{~V}$ (peak-to-peak) and $1.2 \mathrm{MHz}$.

equations of motion in the trap. Since no dc voltage is applied, only the function for $q_{z}$ need be considered:

$$
m / z=\left(1 / q_{z}\right)\left(2 e V / r^{2} \omega^{2}\right)
$$

In eq $1, m / z$ is the mass-to-charge ratio, $V$ is the voltage (peak-to-peak), $\omega$ is the frequency, $r$ is the ring electrode radius, and $e$ is the electron charge.

This relationship was partially studied in our system by observing changes in the spectrum of PFTBA as a function of the rf voltage as shown in Figure 6a-f. In these experiments, the dimensions of the source and the rf were invariant. An estimate of the value of $q_{z}$ at the low mass cutoff was obtained by observing the beginning of attenuation of $m / z 69$ at $V=800 \mathrm{~V}$. From eq 1 , a boundary value of $q_{z}$ for the low-mass cutoff was calculated to be about -0.84 . The high-mass cutoff for zero dc voltage is not predicted by the theoretical stability diagram; rather it is caused by decreasing depth of the potential well as $q_{z}$ decreases below $\sim-0.7$ or the mass-to-charge ratio increases. In our device, the practical high-mass limit is between seven and eight times the low-mass cutoff. The use of ion cooling in normal ion trap mass spectrometers results in a larger mass range for these devices. However, the mass entrapment range of the segmented ring trap is sufficient without cooling for most gas chromatographic applications. We believe a still wider mass range can be obtained by optimization of the physical dimensions and electrical parameters or, if necessary,
6
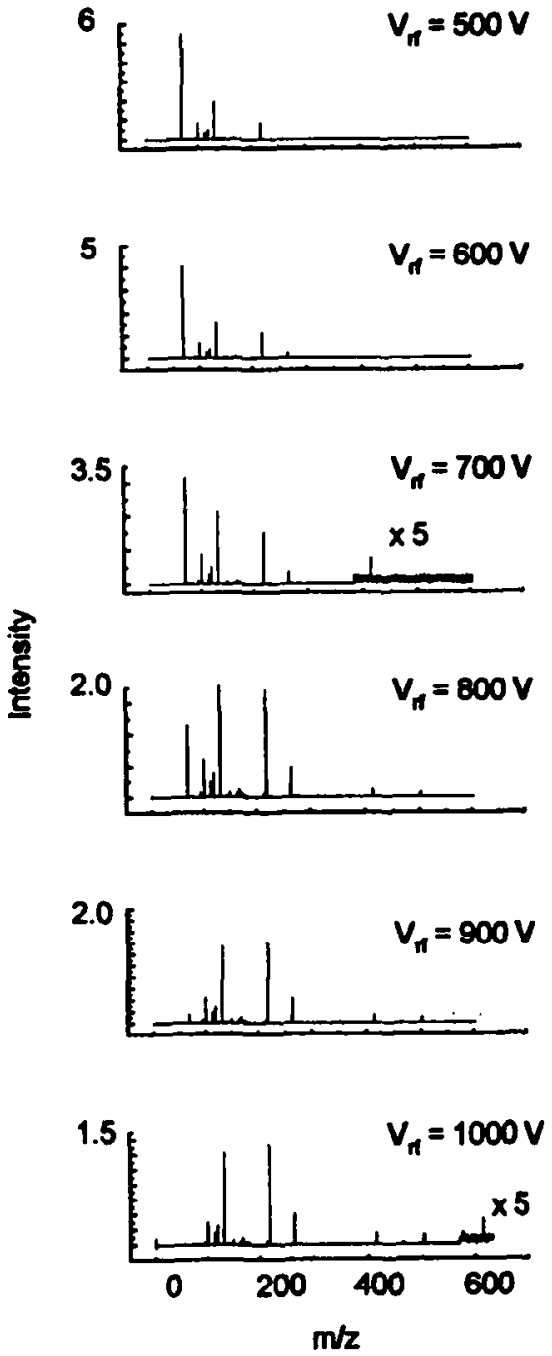

Figure 6. Mass spectra of PFTBA taken with various if voltages on the ion trap. As the rf voltage is increased, increases in both low-mass and high-mass cutoffs occur.

by switching between values of $V$ to obtain low- and high-mass ends of the spectrum alternately.

A further study of the dynamics of the increases in ion population in the trap as a function of ionization time was undertaken. In this case PFTBA was chosen for the analyte so that a wide range of mass-to-charge ratio values would be represented. Figure 7 shows the abundance of the major ions of PFTBA as a function of ionization time. The curves represented by different symbols are actually data points for ions with different mass-to-charge ratio values. The population growth curves follow a first order loss against a constant rate of generation according to the equation

$$
d N / d t=k_{f} p-k_{l} N
$$

where $N$ is the ion abundance, $k_{f}$ and $k_{l}$ are the formation and loss rate constants, and $p$ is the sample pressure. The experimental data points in the first $100-\mu$ s ionization time in Figure 7 are fitted to the 


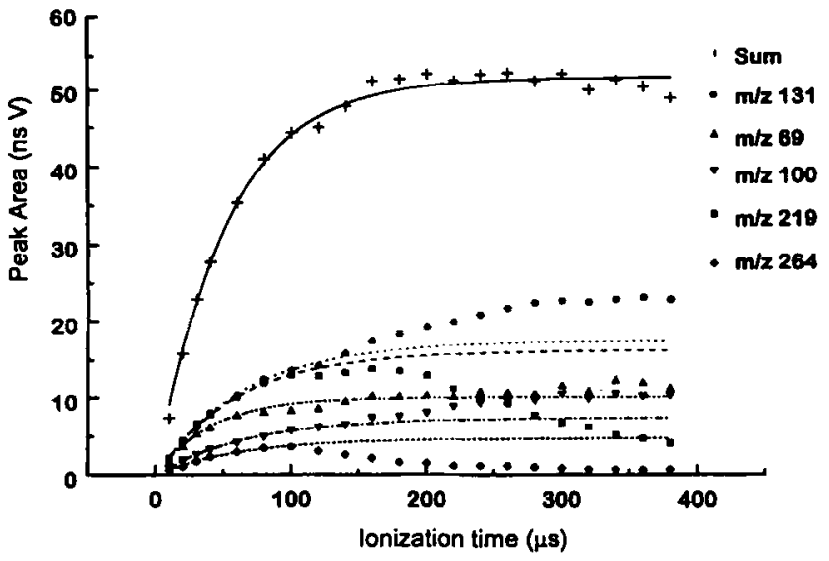

Figure 7. The abundance of the major ions of PFTBA as a function of ionization time (separate points). The smooth lines represent the growth curves fitted to the data points in the first $100-\mu s$ ionization time using eq 3.

integrated form of eq 3 :

$$
N=\frac{k_{f}}{k_{l}} p\left(1-\exp \left(-k_{l} t\right)\right)
$$

The goodness of this fit confirms that a constant rate of ion formation is countered by a loss mechanism whose rate is proportional to the total number of ions in the trap. The time constants for the resulting best-fit exponential growth curves of the different mass-to charge ratio values $1 / k_{1}$ are from 40 to $65 \mu \mathrm{s}$. The source appears to reach steady-state ion abundance at approximately $200 \mu \mathrm{s}$. Also apparent from the data in Figure 7 is that few of the ion abundances fit the exponential growth curve after the first $100 \mu \mathrm{s}$. The abundances of the $m / z 264$ and 219 ions diminish, whereas those of $m / z 100$ and 131 increase. The total abundance, however, remains approximately constant. One possible explanation for this is that ion loss through charge repulsion would be greater for the ions with higher mass-to-charge ratio. Further studies could help elucidate this mechanism, but for now it is important to note that if extraction is accomplished within the first $100 \mu \mathrm{s}$ of ionization, the relative ion abundances remain essentially constant and are characteristic of the sample molecule.

\section{Time-of-Flight as a Mass Analyzer for Trapped Ions}

One measure of the merit of time-of-flight mass analysis is the resolution obtainable over the mass range being measured. For time-array detection, adequate resolution must be obtained over the entire mass range that populates each extraction. In this study, the resolution is determined by the uniformity of the extraction field, the magnitude of the extraction and accelerating fields, the phase relationship between these fields and the rf field, and the energy focusing ability of the ion reflectron and the total flight path. Figure 8 is the mass spectrum of PFTBA obtained with this system.

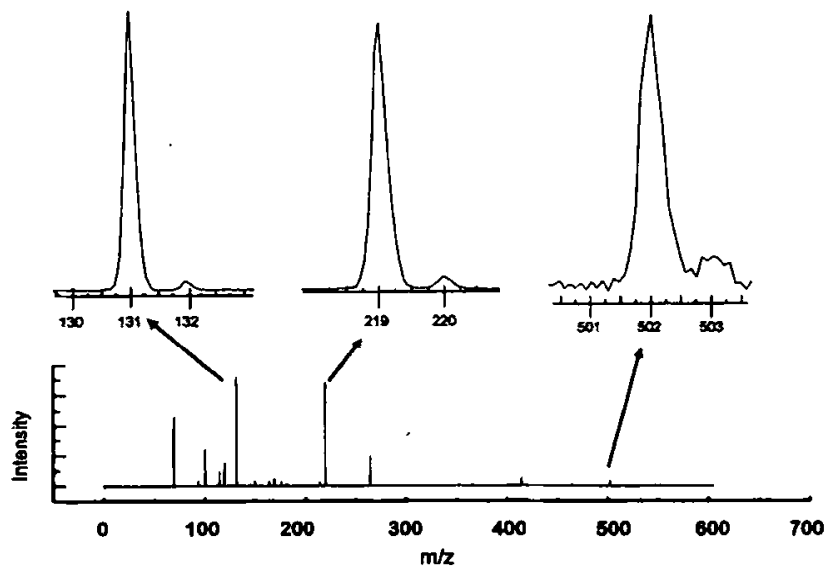

Figure 8. The mass spectrum of PFTBA obtained at $V_{\mathrm{rt}}=800 \mathrm{~V}$ (peak-to-peak), $f_{\mathrm{rf}}=1.2 \mathrm{MHz}$. The insets illustrate the resolution at three points along the mass axis at $m / z 131,219$, and 502 .

Three regions of the mass spectrum are expanded to show the mass resolution obtained in each area. In every instance, the resolution reaches or exceeds that necessary for unit resolution.

The effect of the ionization energy on the observed trapped ions was investigated using chlorobenzene as the analyte. The peak heights of the major ions in the spectrum as a function of the voltage difference between the filament and the electron entry electrode are shown in Figure 9a. In the ion trap, the filament bias voltage is not a direct measure of the ionizing electron energy because of the large effect of the rf field on the electron trajectories. However, the filament bias volt-
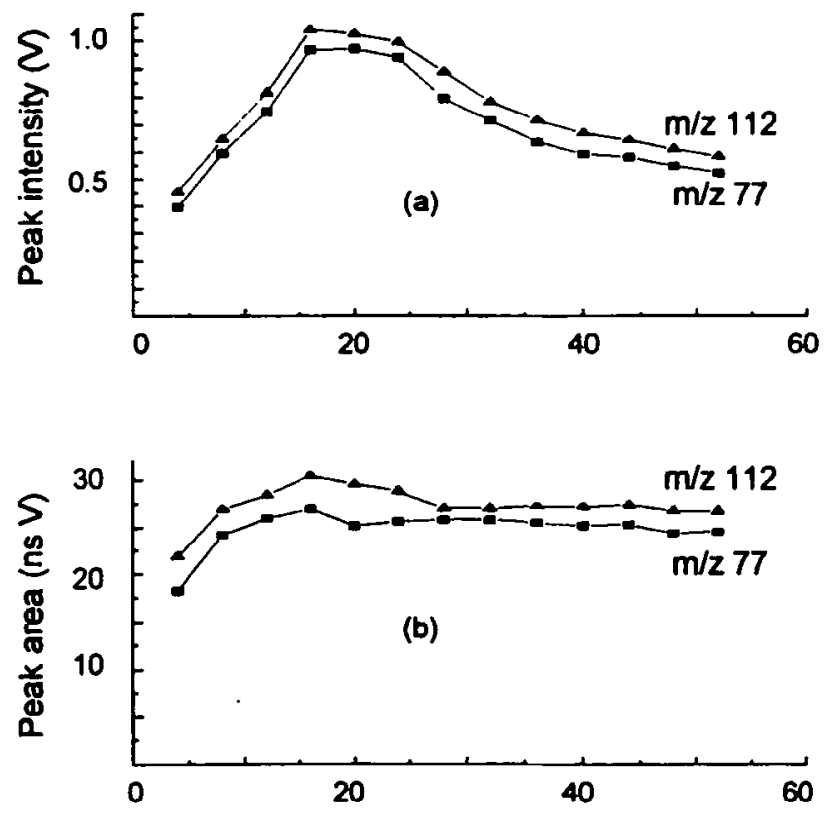

Bias voltage of filament $(M)$

Figure 9. The effect of filament bias voltage on the peaks heights (a) and the peak areas (b) of the major ions of chlorobenzene. 
age is a measure of the energy the electrons possess when they first encounter the rf field, and it is reasonable to assume that the more energy the electrons have at this point, the more energy, on the average, the electrons will possess throughout their trap excursions. The initial slope of this response is similar to that observed for other types of mass spectrometers where sufficient energy for effective ionization must be present. The maximum is reached at lower applied energies than with other sources, undoubtedly due to the accelerative effects of the rf fields. The negative slope at the higher energies is interesting because the trap should not lose these ions. When the peak areas are plotted, as in Figure $9 \mathrm{~b}$, the apparent decrease in abundance at the higher filament bias voltages largely disappears. This is an indication that, at higher bias voltages, it is the temporal focus at the detector that is suffering, not the ion abundance.

\section{Extraction Efficiencies as a Function of Radiofrequency Phase}

At the time of extraction, the rf voltage is turned off and the extraction pulse is applied. In the following series of experiments, the effect of the phase of the $\mathrm{rf}$ field at the time of extraction on the sensitivity and resolution of the TOF analyzer was studied. The points along the applied rf field sampled in this study are indicated in Figure 10. The results of the study are shown in Table 1.

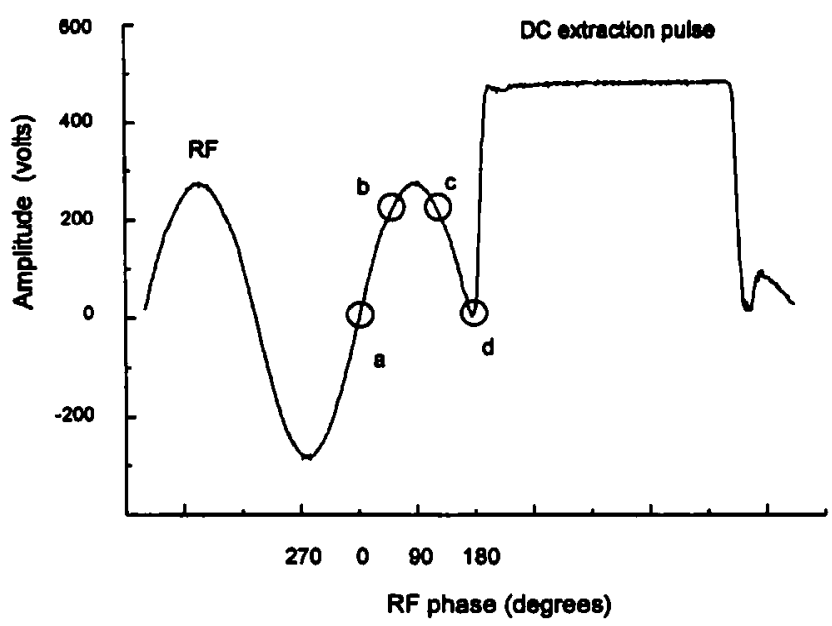

Figure 10. Synchronization of the extraction voltage pulse with the phase of the if voltage. (d) The extraction pulse at phase position $180^{\circ}$. Other phase positions examined were (a) $0^{\circ}$, (b) $80^{\circ}$, and (c) $100^{\circ}$.
The data in this table clearly document the importance of the moment of extraction relative to the phase of the rf field. Let us take as a model case the situation where most of the ions are formed at the $90^{\circ}$ angle, which is the point of maximum electron energy and density in the trap, and consider the force effects of the field relative to a single rf cycle only: at the phase point of $0^{\circ}$, the ions move with minimum acceleration (actually, none) and relative maximum velocity (if the field has been sensed in the last half cycle) in a direction opposite to the extraction axis. However, the direction of the instantly applied extraction field is the same as that of the rf field. At the $80^{\circ}$ moment, the ions in the trap respond to a near maximum acceleration toward the extraction axis, which reduces the relative velocity in the opposite direction to near zero, and the If field is about to change its sign. In both of these situations, the resolution and sensitivity are enhanced. What these two phase points have in common is the direction of change of the applied extraction field and the instantaneous rf field. At the $100^{\circ}$ position, the ions begin to move in directions toward the exit cap, while the relative magnitude and direction of the acceleration vector are approximately the same as at the $80^{\circ}$ point, the application of the extraction voltage reverses the field. Extraction at this point results in attenuation of both resolution and sensitivity. At the $180^{\circ}$ position, the ions move in the direction of the extraction axis at their maximum relative velocity, again the applied extraction voltage is opposite in direction of change to the rf voltage and both the sensitivity and resolution are diminished. The significant attenuation in detected peak areas at these two positions means that many of the trapped ions simply are lost in the extraction and measurement processes. In all of these situations, the amount of ion acceleration and relative velocity appear to have little effect on the quality of the resulting TOF analyses. Only in cases where the direction of the changing field is reversed upon application of the extraction pulse do we observe significant attenuation, and the greater the change, the greater the loss. By some means not yet clear, this action may spray or scatter the ions from the source during the finite transition time of the changing opposing fields. With regard to the advantageous cases, the similarity of the results at 0 and $80^{\circ}$ suggests that optimum performance may be achieved with a considerable leeway along the phase axis from 0 to $80^{\circ}$.

Overall instrument performance for sensitivity and dynamic range was tested by obtaining a working

Table 1. Effects of the timing of the extraction pulse in relation to the rf phase

\begin{tabular}{cccc}
\hline & \multicolumn{3}{c}{ Height/resolution } \\
\cline { 2 - 4 } f phase & $m / z 69$ & $m / z 131$ & $m / z 219$ \\
\hline \hline 0 & $40,489 / 756$ & $16,702 / 857$ & $11,353 / 1,019$ \\
80 & $41,510 / 663$ & $16,760 / 772$ & $11,415 / 986$ \\
100 & $15,370 / 541$ & $4,815 / 562$ & $2,936 / 767$ \\
180 & $5,394 / 359$ & $1,995 / 412$ & $1,378 / 645$ \\
\hline
\end{tabular}




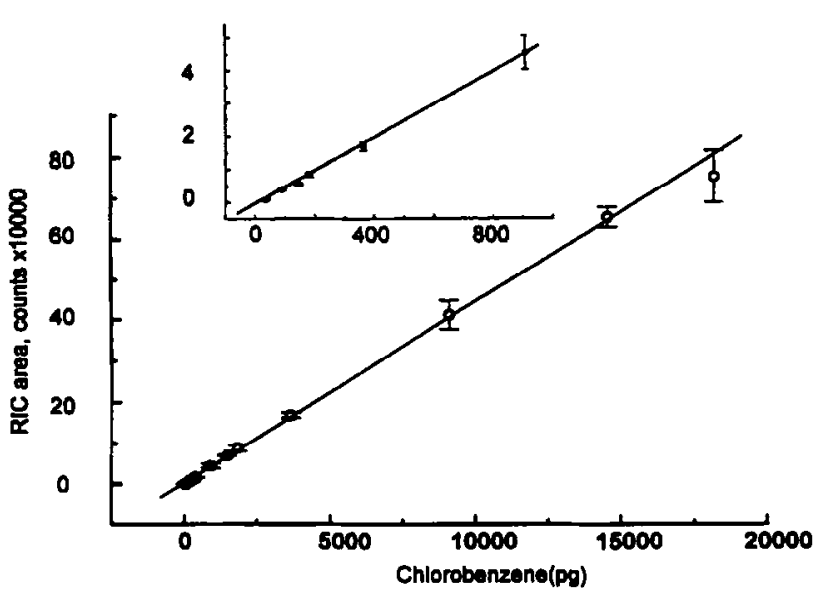

Figure 11. Working curve for chlorobenzene that illustrates the linearity of response over nearly 3 orders of magnitude.

curve as shown in Figure 11. The analyte for this study was chlorobenzene. A volume of $1 \mu \mathrm{L}$ of chlorobenzene in the hexane solvent was injected into the gas chromatograph operated in the split injection mode. Analyte concentrations varied from $20 \mathrm{ng} / \mu \mathrm{L}$ to $10 \mu \mathrm{g} / \mu \mathrm{L}$. With a split ratio of 1:550, a linear range was confirmed from $36 \mathrm{pg}$ to $18 \mathrm{ng}$. Figure 12 shows the reconstructed ion chromatogram from $\mathrm{m} / \mathrm{z} 112$ of the 36-pg sample injection. A mass peak-finding technique was used to differentiate the signal peak and background noise in the individual mass spectra.

In Figure 13 the mass spectrum of chlorobenzene taken at scan 34 is shown. The voltage across each MCP plate of the detector is $980 \mathrm{~V}$. A signal-to-background noise (peak-to-peak) ratio of 20:1 is obtained for this 36-pg sample at a spectral generation rate of 4 spectra per second. These data were collected with an ionization time of $100 \mu \mathrm{s}$ and an extraction rate of $1000 \mathrm{~Hz}$. Each mass spectrum is the sum of 250 TOF transients. They verify the earlier assumption that at $100-\mu$ s ionization time, the trapped ion population is a valid measure of the amount of sample that is introduced into the source. Better results could be obtained with an extraction rate of $6 \mathrm{kHz}$, which is the recipro$\mathrm{cal}$ of the maximum ion flight time. In our current system, the maximum extraction frequency is limited by the nature of the $\mathrm{rf}$ generator and the transient recorder used and not by the ion storage, extraction, and focusing optics. Achievement of the maximum extraction rate of $6 \mathrm{kHz}$ would provide the same signal-to-noise ratio obtained for the $36 \mathrm{pg}$ of chlorobenzene in Figure 12, but at 24 spectra per second. Conversely, the better sensitivity obtained with the $6-\mathrm{kHz}$ extraction at a lower number of spectra per second will improve ion statistics at the limit of detection, which increases the linear range of measurement.

\section{Conclusions}

The segmented ring trap with its cylindrical geometry exhibits the ability to trap ions and hold them indefi-

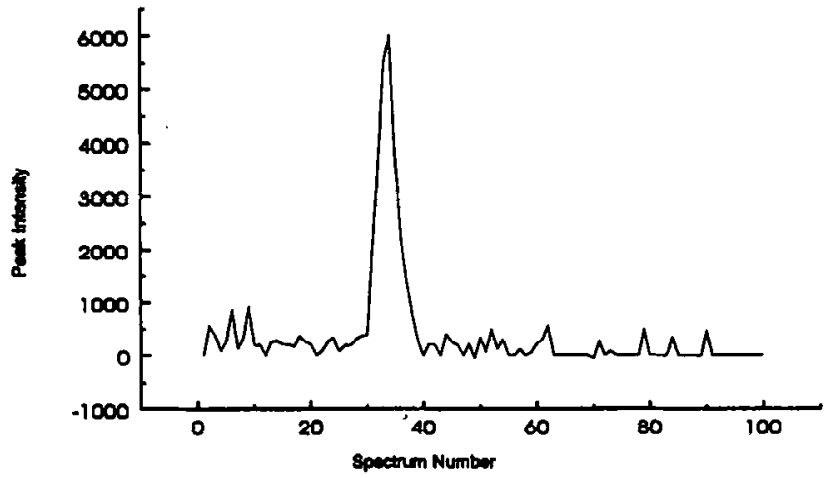

Figure 12. Reconstructed ion chromatogram (RIC) for $m / z=$ 112 for the $36 \mathrm{pg}$ of chlorobenzene detected. Due to the noise near the detection limit, a peak-finding technique (threshold = 50) was used.

nitely. The mass range of entrapment closely follows the expected stability diagram for the cylindrical ion trap, which, under the parameters being used, closely follows the classical quadrupole equations as well. The fields are surely not perfectly quadrupolar, but perfectly quadrupolar fields are not required for broad range ion storage, only for selective mass-to-charge ratio value rejection.

The uniform extraction fields possible with this geometry enable good mass resolution to be achieved without the need for ion cooling prior to mass analysis. The resulting SRS-TOF mass analysis combined with time array detection is providing the hoped-for speed, sensitivity, and quality of spectral data. Without component optimization, the present unit readily generates information for up to 1000 spectra per second. As shown in Figures 5 and 7, population growth is still in the nearly linear phase at a $100-\mu \mathrm{s}$ ionization and storage time. Analytically reliable linear growth to this point is confirmed by the data in Figure 8, where a series of varying analyte concentrations show a linear

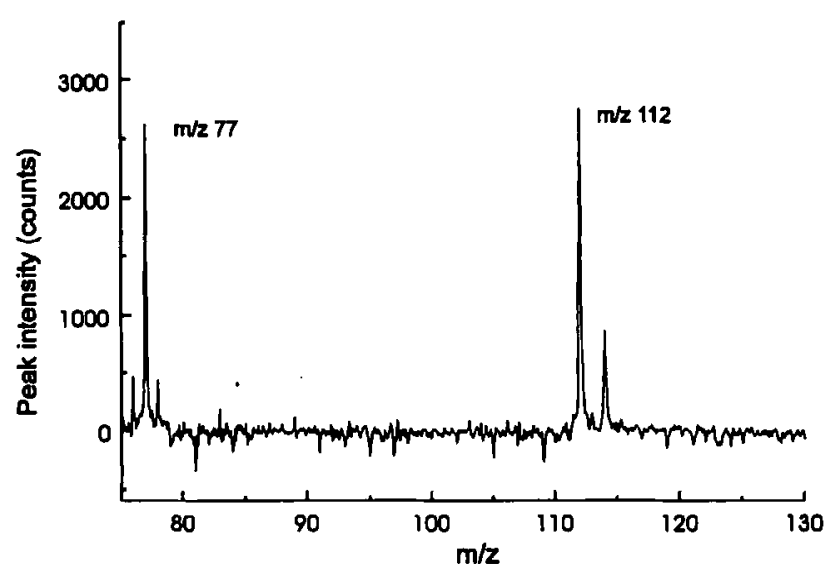

Figure 13. Mass spectrum of scan 34 from the RIC of Figure 12 with background subtraction and fast Fourier transform filter smoothing technique. This spectrum is obtained by summing 256 transients. 
response of ion population to concentration with this ionization timing.

With a $100-\mu$ s ionization time and a $1-\mathrm{kHz}$ extraction rate, the sample utilization duty cycle is very nearly $10 \%$. With improved electronics and adjustments to the source design, one can realistically envision a sample duty-cycle efficiency approaching $100 \%$ for continuous chromatographic applications. A high ion compaction value is achieved; all the ions of each mass-to-charge ratio value generated during $100 \mu \mathrm{s}$ arrive at the detector within approximately $80 \mathrm{~ns}$. This gives rise to an instantaneous peak detector current that is 1200 times greater than that produced by the average ion generation rate. In a TOF analyzer optimized for maximum ion transmission, this should result in detection limits better than those of selected ion monitoring, while still providing 100 or more full spectra per second. If so, the segmented ring ion trap source combined with time-of-flight mass analysis and time-array detection could offer substantial advantages in sensitivity and sampling rate for situations where rapid continuous sampling of dynamic events is required.

\section{Acknowledgments}

This work was supported in part by NIH Grant GM49922. The authors also acknowledge helpful discussions with Paul Vlasak and Mary Seeterlin at Michigan State University, Scott McLuckey at Oak Ridge National Laboratory. George Yefchak and Hewlett-
Packard, Inc. provided us with a data acquisition board and software.

\section{References}

1. Holland, J. F.; Enke, C. G.; Allison, J.; Stults, J. T.; Pinkston, J. D.; Newcome, B. N.; Watson, J. T. Anal. Chem. 1983, 55, 997A-1112A.

2. Paul, W.; Steinwedel, H. Z. Naturforsch. 1953, 8a, 448-450.

3. Waldren, R. M.; Todd, J. F. J. Int. J. Mass Spectrom. Ion Phys. 1979, 29, 314-335.

4. (a) Michael, S. M.; Chien, M.; Lubman, D. M. Reo. Sci. Instrum. 1992, 63, 4277-4284; (b) Chien, B. M.; Michael, S. M.; Lubman, D. M. Rapid Commun. Mass Spectrom 1993, 7, 837-843; (c) Chien, B. M.; Michael, S. M.; Lubman, D. M. Anal. Chem. 1993, 65, 1916-1924; (d) Michael, S. M.) Chien, B. M.; Lubman, D. M. Anal. Chem. 1993, 65, 2614-2620; (e) Chien, B. M.; Lubman, D. M. Anal. Chem. 1994, 66 1630-1636.

5. Grix, R.; Gurener, U.; Li, G.; Stroh, H.; Wollnik, H. Int. J. Mass Spectrom. Ion Processes 1989, 93, 323-330.

6. Mamyrin, B. A.; Karataev, V. I.; Shmikk, D. V.; Zagulin, V. A. Sov. Phys.-JETP 1973, 37, 45-48.

7. Langmuir, D. B.; Langmuir, R. V.; Shelton, H.; Wuerker, R. F. U.S. Patent 3065640, 1962.

8. Ji. Q.; Vlasak, P. R.; Holland, J. F.; Enke, C. G. Proceedings of the 42nd ASMS Conference on Mass Spectrometry, Chicago, 1994.

9. Vlasak, P. R.; Beussman, D. J.; Ji, Q.; Enke, C. G. J. Am. Soc. Mass Spectrom., in press.

10. Mather, R. E.; Waldren, R. M.; Todd, J. F. J.; March, R. E. Int. J. Mass Spectrom. Ion Phys. 1980, 33, 201-230. 